\title{
Évaluer les impacts des recherches en agriculture sur la société et les écosystèmes: outils, méthodes, études de cas
}

\author{
Ludovic Temple ${ }^{1,5, *}$, Ariane Gaunand ${ }^{2}$, Gilles Trouche ${ }^{3,5}$ et Eric Vall ${ }^{4,5}$ \\ ${ }^{1}$ Cirad, UMR INNOVATION, 34398 Montpellier, France \\ 2 Inra, Délégation à l'Évaluation, UMR LISIS, 147 rue de l’Université, 75338 Paris, France \\ 3 Cirad, UMR AGAP, 34398 Montpellier, France \\ 4 Cirad, UMR SELMET, 34398 Montpellier, France \\ ${ }^{5}$ Univ Montpellier, 34090 Montpellier, France
}

\begin{abstract}
Résumé - Confrontés aux évolutions des enjeux et modèles de développement touchant les secteurs de l'agriculture et de l'alimentation, les organismes de recherche interrogent les méthodes d'évaluation des impacts liées aux activités de recherche. En effet les cadres méthodologiques conventionnels utilisés depuis les années 1950 ne répondent plus aux attentes sociétales et aux réalités de l'activité de recherche dans ce domaine. Le numéro thématique des Cahiers Agricultures "Évaluer les impacts des recherches en agriculture sur la société: outils, méthodes, études de cas» présente les nouvelles approches méthodologiques proposées ces dernières années par l'INRA, le CIRAD et le CGIAR. Ces méthodes utilisent une démarche commune qui met l'accent sur les dimensions systémiques des processus d'innovation, la dimension multi-niveaux des impacts, la temporalité longue. Elles diffèrent sur certains points, comme par exemple la participation des parties prenantes dans l'évaluation des impacts. Le test empirique de ces démarches dans différentes études de cas structure une partie du numéro thématique dans les pays en développement. Il est enrichi par des analyses transversales aux études de cas ou sur des situations portées par d'autres orientations méthodologiques et dans d'autres contextes géographiques. Certaines contributions identifient les limites des différentes méthodes pour de futurs fronts de recherche. Cet article de synthèse expose des connaissances sur la compréhension des impacts de l'activité de recherche. Il invite à reconsidérer le rôle de la recherche dans les processus d'innovation.
\end{abstract}

Mots clés : recherche agronomique / évaluation d'impact / approche méthodologique / innovation / politique de recherche

\begin{abstract}
Evaluation of the impacts of agricultural research on society and ecosystems: tools, methods, case studies. In light of the evolution of issues and development models in the agriculture and food sector, research organizations are questioning the methods to assess the impact of research activities. Indeed, conventional methodological frameworks used since the 1950s are increasingly less adapted to societal expectations and realities of agricultural research. This thematic issue of Cahiers Agricultures "Evaluation of the impacts of agricultural research on society: tools, methods, case studies" presents new methodological approaches developed in recent years by INRA, CIRAD and CGIAR. These methods build on common theoretical underpinnings that emphasize the systemic dimensions of the innovation process, multiple perspectives and longer temporality of impact generation. They also differ in certain dimensions, such as the level of participation of stakeholders in the assessment of impacts. One part of this thematic issue is dedicated to the empirical test of these approaches in developing countries. The issue is enriched by papers providing cross-cutting analyses of the case studies, or presentation of different methods and geographical contexts. Finally, some articles identify the limitations of these methods for future research. This synthesis article presents knowledge on the understanding of the impacts of research activities, and invites readers to reconsider the role of research in the innovation process.
\end{abstract}

Keywords: agricultural research / impact evaluation / methodology / innovation / research policy

\footnotetext{
*Auteur de correspondance : ludovic.temple@cirad.fr
} 
Les organismes publics de recherche du secteur de l'agriculture et de l'alimentation sont interpellés sur les nouveaux enjeux sociétaux de développement: transition écologique, réduction des inégalités sociales, sécurité alimentaire, changement climatique, révolutions technologiques (Touzard, 2018). La prise en compte de ces enjeux se complexifie avec la diversification des parties prenantes, la globalisation numérique (informations et connaissances) et la demande de démonstration croissante de l'utilité sociale des actions de la recherche exprimée par les partenaires techniques, financiers et socio-politiques de la recherche. Ces multiples interpellations rendent nécessaires la construction de méthodes pour comprendre et évaluer les impacts «au sens large» (en référence au champ de littérature sur le «broader impact») (Bornmann, 2013). Ces impacts englobent les dimensions économiques, culturelles, sanitaires, environnementales, organisationnelles, sociales et politiques des retombées de la recherche (Joly et al., 2015a, b) et peuvent potentiellement affecter des entités variées de notre société et de notre écosystème.

Depuis les années 1950, les méthodes d'évaluation d'impact mobilisées par les institutions de recherche intervenant dans l'agriculture et l'alimentation dérivent des méthodes économiques d'évaluation des politiques publiques (Berriet et al., 2014). Selon ces méthodes, l'impact de la recherche est établi par le calcul du taux de rentabilité des investissements de recherche. Ces méthodes sont aujourd'hui remises en débat, en raison de leurs insuffisances à rendre compte des impacts indirects de la recherche tels que les externalités (sociales, environnementales, économiques), les effets rétroactifs de la connaissance sur les trajectoires technologiques, parfois qualifiés de «débordements», spillovers, les changements structurels à long terme, les risques environnementaux et sociaux (Weißhuhn et al., 2017). Elles sont aussi remises en question pour leur capacité insuffisante à expliciter la satisfaction des attentes sociétales dans leur diversité (Temple et al., 2016).

Dans ce contexte ont émergé de nouvelles méthodes d'évaluation de l'impact de la recherche en agriculture et en alimentation sur la société et les écosystèmes, en mettant l'accent sur: (i) la multi-dimensionnalité de l'impact de la recherche (Joly et al., 2015a, b); (ii) la temporalité et la sérendipité des chemins d'impacts (Ruegg et Feller, 2003); (iii) la complémentarité entre des approches qualitatives et quantitatives de caractérisation des impacts (Donovan, 2011; Naudet et al., 2012); (iv) la compréhension de la dimension systémique des processus d'innovation (Spaapen et van Drooge, 2011; Mayne, 2012; Bornmann, 2013; Temple et al., 2016; Douthwaite et al., 2017); enfin (v) la participation des acteurs de l'innovation dans le processus d'évaluation (Wiek et al., 2014). La métaphore de «chemins d'impact» au centre de ce renouvellement de méthodes repose en soi sur deux idées principales : i) un enchaînement de causalité entre les inputs (moyens et connaissances) mobilisés pour mettre en œuvre la recherche, les produits et résultats de cette recherche (outputs), les changements liés à l'appropriation des résultats (que nous qualifieront par la suite fréquemment d'outcomes) et les impacts sur des indicateurs de développement et ii) la diversité de ces enchaînements qui interagissent au sein d'un même processus d'innovation.
Le numéro thématique des Cahiers Agricultures «Évaluer les impacts des recherches en agriculture sur la société : outils, méthodes, études de cas » (Gaunand et al., 2018) présente ainsi différents exemples du renouvellement des méthodes d'évaluation d'impact développées par différentes institutions de recherche publique et mises en application dans divers contextes agricoles (Renkow et Byerlee, 2010; Joly et al., 2015a, b; Temple et al., 2018).

Le secteur de l'agriculture et de l'alimentation comporte en effet des spécificités du fait de la nature biologique des processus, de sa dépendance aux aléas environnementaux, des déterminants économiques, sociaux et culturels de ses activités et enfin de la spécificité localisée des écosystèmes, qui créent une diversité de contraintes et de ressources pour l'innovation (Touzard et al., 2015). Ainsi, il convient de considérer les impacts sur des périodes longues et à des échelles variées (parcelle, exploitation agricole ou entreprise, système de production, territoire, filière, économie nationale). À cet effet, plusieurs types de contributions complémentaires sont mobilisés à partir de travaux menés depuis 2010 dans différentes institutions de recherche agricole (Cgiar, Cirad, Inra, Ird).

Un premier ensemble d'articles présente des études de cas utilisant la méthode d'évaluation participative proposée par le Cirad et nommée ImpresS (Barret et al., 2017). Cette méthode renvoie à un ensemble complémentaire d'outils d'analyse et de principes qui ont été testés et améliorés au fil de la conduite de ces études de cas. Ces dernières, conduites par des chercheurs du Cirad et leurs partenaires scientifiques du Sud, montrent comment la diversité des contextes géographiques, socioculturels et institutionnels influence les mécanismes d'interaction entre la recherche et le développement ainsi que la nature des impacts générés. Elles illustrent également la variété des rôles des chercheurs dans les chemins d'impact. Elles soulignent enfin la diversité des résultats de la recherche, et notamment les caractéristiques de ses outcomes (changements de pratiques et de modes d'organisation) en termes de nature, d'intensité et de fonctions, ainsi que leurs interactions avec d'autres facteurs, autrement dit de résultats qui vont bien audelà de la production de connaissances scientifiques ou techniques.

Ainsi, l'article de Breumier et al. (2018), qui étudie les impacts de 30 années de sélection variétale du riz pluvial d'altitude à Madagascar, présente un chemin d'impact avec de nombreux outputs (résultats scientifiques et techniques), et trois outcomes pour lesquels l'accompagnement de la recherche a été important: des réseaux d'expérimentation variétale et de champs de démonstration en milieu paysan, une organisation formalisée de la production de semences et un partenariat recherche-vulgarisation de longue durée. De ces outcomes résultent une large adoption des nouvelles variétés de riz développées pour ces Terres Hautes, et des impacts en cascade comme la réduction de l'insécurité alimentaire des ménages, l'augmentation des revenus des producteurs, des transformateurs et des commerçants, la diversification des activités agricoles et la capitalisation dans l'élevage, et au final «une plus grande tranquillité d'esprit» pour les familles rurales, à condition d'investir dans la fertilisation des champs rizicoles pour garantir la durabilité de ce système agricole innovant. 
L'article de Clavel et Gaye (2018) décrit la contribution de la recherche à l'organisation d'une filière nationale de production de semences d'arachide de qualité certifiée, approvisionnée par une trentaine de coopératives, et sécurisée par un système contractuel de commercialisation avec le Crédit agricole du Sénégal. Dans le chemin d'impact, les auteurs identifient plusieurs facteurs clés qui ont contribué à ce succès (i) la conduite d'une opération pilote de recherche et développement; (ii) un partenariat diversifié avec la filière; et (iii) la professionnalisation des producteurs. Les auteurs identifient aussi un risque (donc un impact potentiellement négatif) de fragilisation des coopératives si l'expertise semencière développée se retrouvait mobilisée à des fins purement commerciales.

L'article de Cerdan et al. (2018) analyse la diversité des rôles de la recherche au cours de dix années de travail consacrées au développement d'une Indication géographique (IG) pour un vin au Brésil. Au départ, l'activité de la recherche était concentrée sur la production de connaissances (procédure, techniques) pour délimiter l'aire d'appellation, et sur l'obtention de financements pour répondre aux sollicitations des organisations de producteurs. Puis son rôle s'est élargi à la formation des acteurs porteurs de l'IG, sur les questions de structuration de l'aire territoriale et de valorisation de l'appellation. Enfin, la recherche a contribué à la reconnaissance de l'IG auprès des institutions étatiques en structurant différentes formes de réseaux. Ce travail met aussi en exergue le rôle de la recherche dans le renforcement des capacités réflexives des acteurs durant la conception de projets de développement territoriaux.

L'article de Ferré et al. (2018) souligne comment un partenariat entre chercheurs et équipementiers, guidé par les besoins exprimés par les utilisateurs, les transformatrices de fonio (diminuer la pénibilité du travail de décorticage et blanchiment des graines), a permis de transférer des connaissances et de contribuer aux apprentissages nécessaires pour la diffusion de nouveaux équipements de transformation du fonio au Burkina Faso et au Mali.

Enfin, l'article de Queste et Wassenaar (2018), analysant les impacts d'un projet de recyclage des déchets organiques à la Réunion, met en évidence l'effet des processus de concertation entre chercheurs, agents techniques et décideurs politiques. Cette concertation aboutit à (i) la reformulation collective des problèmes; (ii) l'émergence et la dissémination de savoir-faire; (iii) l'amélioration de l'organisation de la filière de recyclage. Elle fait émerger une filière intégrée de recyclage des déchets organiques transformés en fertilisants normés, utilisés sur la culture de la canne à sucre.

Ces études de cas, réalisées dans des situations géographiques et institutionnelles contrastées, et sur des innovations de natures différentes (technologique ou organisationnelle), illustrent la diversité et l'évolution des rôles des chercheurs dans la construction des chemins d'impact. Ainsi, Cerdan et al. mettent en avant l'importance de la crédibilité conférée à l'organisme de recherche qui inspire confiance aux viticulteurs. Queste et Wassenaar identifient quant à eux l'appui des chercheurs pour la reformulation collective des problèmes et l'apprentissage comme étant des rôles clés de la recherche dans le processus de génération d'impact sociétaux. Enfin, Breumier et al. et Cerdan et al. notent que les rôles et fonctions des chercheurs évoluent au cours des chemins d'impact. De manière générale, ces études de cas soulignent que les rôles des chercheurs ne se limitent pas à la production de connaissances, mais concernent aussi le renforcement de capacités et les apprentissages (individuels et collectifs) des acteurs, qui sont des ressources socialement re-mobilisables en dehors du secteur spécifique de l'agriculture.

Enfin, ces études de cas, en illustrant que les impacts touchent des bénéficiaires variés dans des sphères plus ou moins distantes des utilisateurs directs des résultats de recherche, montrent que la causalité entre l'activité de recherche et la transformation sociétale résulte d'une coordination systémique de différentes ressources cognitives, informationnelles et d'apprentissage (Boutillier et Laperche, 2016; Temple et al., 2018). Les organismes de recherche sont ici interpellés pour assumer la diversité des rôles et des implications des chercheurs pour structurer des systèmes d'innovation efficaces dans leurs impacts sur le développement.

Ce corpus d'études de cas permet aussi de prendre du recul sur la mise en œuvre de la méthode ImpresS. Deux éléments conduisent les porteurs de ces études à spécifier les modalités d'usage de cette méthode: la nature du processus d'innovation étudié, et l'intensité de la participation des acteurs au processus même d'évaluation. D'une part, selon que la recherche est «initiatrice» de la nouveauté (cas des nouvelles variétés ou équipements) ou qu'elle se positionne en accompagnement et renforcement des conditions de mise en œuvre de changements institutionnels ou technologiques (cas des IG et du recyclage de déchets), les outils méthodologiques d'ImpresS sont utilisés différemment. D'autre part, selon la nature des ressources humaines mobilisées (compétences et spécialités scientifiques, institutions représentées, importance de l'engagement des porteurs du Sud...) et les difficultés inhérentes aux terrains d'enquête (accès aux données secondaires, logistique, traduction, disponibilité et motivation des partenaires), la participation effective des parties prenantes à l'évaluation a été variable. La méthode ImpresS a donc été appliquée de manière relativement hétérogène, ce qui a été source d'amélioration continue du cadre commun d'analyse, mais limite aussi la puissance de l'analyse transversale.

Par ailleurs, ces études de cas révèlent que la démarche ImpresS permet de dépasser deux obstacles dans l'évaluation de l'impact, spécifiques au contexte de développement des pays du Sud. Premièrement, la faible disponibilité de bases de données, pour laquelle la méthode ImpresS prévoit la génération des données nécessaires. Deuxièmement, une fragilité d'organisation de la société civile et des organisations professionnelles agricoles, donc une faible capacité d'expression de la demande sociale, qui doit être accompagnée dans sa construction. Pour y répondre, la démarche ImpresS propose une approche multi-acteurs participative.

Un deuxième ensemble de contributions présente une analyse transversale de l'ensemble des 13 études de cas conduites en 2015-2016.

Toillier et al. (2018) focalisent leur travail sur le renforcement des capacités à innover induit par la recherche. Les auteurs identifient quatre postures permettant de favoriser le renforcement des capacités des acteurs: i) faciliter des apprentissages de façon non supervisée, ii) planifier et encadrer des apprentissages, iii) créer des besoins d'apprentissage et y répondre pas-à-pas et iv) se laisser guider par l'exploration et les besoins des utilisateurs. La pratique même de l'évaluation des relations entre la recherche et le développement est alors un 
outil en soi, qui génère des apprentissages nécessaires au bon pilotage de l'innovation pour le développement.

Dabat et Grandjean (2018) caractérisent la contribution des acteurs des politiques publiques dans la réalisation des chemins d'impact en analysant ces mêmes 13 études de cas. Les auteurs mettent en évidence les stratégies d'action des acteurs publics, et montrent comment le contenu des politiques publiques peut créer les conditions institutionnelles à l'adoption des innovations et la production d'impacts. Ils soulignent, cependant, malgré le repérage d'inflexions dans l'agenda politique des différents pays, la faible réceptivité des acteurs publics aux résultats de la recherche.

Enfin, un troisième ensemble de contributions propose d'autres approches méthodologiques pour l'analyse et l'évaluation de l'impact.

Dans le contexte de l'évolution des riziculteurs camarguais vers l'agriculture biologique, Quiédeville et al. (2018) utilisent la méthode de Social Network Analysis (SNA) pour approfondir la compréhension des liens entre tous les acteurs du système d'innovation et l'évolution de leur influence respective dans le temps en fonction des projets de recherche mis en œuvre (Wasserman, 1994).

Blundo-Canto et al. (2017) proposent la mise en place d'un suivi-évaluation systématique des résultats de la recherche, c'est-à-dire plus spécifiquement focalisé sur les outcomes, avec une approche qualifiée par les auteurs de «récolte des outcomes ». Ce suivi-évaluation vise à caractériser notamment les apprentissages pour adapter et améliorer les processus d'innovation. Cette démarche permet de générer des apprentissages collectifs, portant sur les raisons et les façons d'utiliser les résultats de recherche et sur la détection d'outcomes imprévus.

L'article de Colinet et al. (2017) propose une méthode pour établir des barèmes génériques de mesure des principales dimensions d'impact des recherches, sur la base de descripteurs locaux exprimés par les bénéficiaires. Les auteurs illustrent la méthode de construction de tels barèmes par une application sur la mesure des impacts politiques des recherches de l'Inra.

Enfin, Minkoua et Temple (2018) présentent une méthode d'analyse de l'adéquation de l'offre et de la demande de recherche, basée sur l'utilisation de données secondaires (indicateurs de besoins des chefs d'entreprises et publications scientifiques). Ils soulignent le "désalignement» de l'offre de recherche par rapport aux demandes du secteur entrepreneurial. Ils concluent au besoin d'améliorer la production de statistiques sur l'innovation, et notamment d'indicateurs macro-économiques, pour améliorer l'analyse de l'impact de la recherche.

La diversité des environnements politiques et institutionnels de la recherche publique et des organismes de développement souligne le besoin de proposer des démarches méthodologiques flexibles, adaptables à ces différents contextes. Bien que les impacts résultent de la production de connaissances qualifiées d'utiles (Minkoua et Temple, 2018), la réalisation de ces impacts reste tributaire de contextes et d'agendas totalement extérieurs aux acteurs de la recherche (Queste et Wassenaar, 2018). Ils sont aussi parfois inattendus comme le montrent Blundo-Canto et al. (2017). Dans presque toutes les situations mises en œuvre, la compréhension des mécanismes (ex: structuration de filières, renforcement de capacités, concertation, évolution réglementaire...) expliquent les relations de causalité entre l'activité de recherche, la genèse de l'innovation et sa mise en œuvre dans le système social et les écosystèmes. Cette compréhension repose sur des travaux faisant interagir les sciences humaines et sociales avec les sciences agronomiques ou biologiques.

Les études de cas et les méthodes présentées dans ce numéro thématique mettent en évidence la complémentarité des recherches portant sur la compréhension des interactions entre les dimensions technique, institutionnelle et organisationnelle de l'innovation. Cette complémentarité génère des capacités d'apprentissage collectives sur la création d'impacts qui sont nécessaires pour mobiliser les connaissances nouvelles comme moteurs du développement (Stiglitz et Greenwald, 2014).

De nombreux enjeux méthodologiques restent cependant à surmonter. Le principal porte sur la difficulté à mesurer ou à quantifier une causalité entre l'activité de recherche et un impact, dans une démarche compréhensive de processus complexe. Ce processus fait interagir des combinaisons différentes d'acteurs selon les «phases» de l'innovation (conception, émergence, mise en œuvre -implémentation-, dissémination) qui sous-tendent les chemins d'impact. Une seconde difficulté porte sur la connaissance des trajectoires d'innovation infructueuses, des risques et des impacts négatifs inhérents à tout changement, tel que l'accroissement des inégalités sociales, la concentration des firmes ou l'intégration des filières, qui peuvent exclure des populations de l'accès à des ressources «qualifiables» dans certaines situations de biens communs: patrimoine génétique des plantes, eau, biomasse (Stiglitz et Greenwald, 2014; Godin et Vinck, 2017 ; Giraud, 2018) et que certaines études de cas identifient. Des développements méthodologiques devraient permettre de mieux tirer parti des leçons à tirer de ces échecs pour orienter les actions futures. Ces limites montrent la nécessité d'améliorer les démarches d'évaluation de l'impact, pour analyser aussi les externalités négatives potentielles, qu'elles soient sociétales, économiques ou environnementales.

Au-delà de ces limites, les différentes démarches d'évaluation présentées montrent que la participation des acteurs, même partielle ou imparfaite, permet de mettre en lumière des impacts non anticipés, que ce soit par la recherche ou par les bailleurs de fond. Ces effets de « débordement» sont généralement peu référencés dans les démarches d'évaluation classiques, alors qu'ils sont le plus souvent révélateurs d'un effet de renforcement des capacités des acteurs, induit, au moins en partie, par la recherche.

Ces démarches d'évaluation de l'impact de la recherche questionnent aussi de manière réflexive les modèles d'innovation mobilisés. Elles interrogent la communauté scientifique sur les processus permettant de générer une connaissance scientifique utilisable et accessible pour des acteurs de terrain, mais aussi sur les conséquences des stratégies d'action ou d'investissement de ces acteurs en termes d'équité entre les générations, entre les territoires et entre les populations du point de vue de l'amélioration des conditions de vie.

\section{Références}

Barret D, Blundo Canto G, Dabat M-H, Devaux-Spatarakis A, Faure G, Hainzelin E, et al. 2017. Guide méthodologique ImpresS : Évaluation ex post des impacts de la recherche agronomique dans les pays du Sud. Montpellier (France): CIRAD, 96 p. DOI: 10.19182/agritrop/00005. 
Berriet M-H., Labarthe P, Laurent P-C. 2014. Empirical validity of the evaluation of public policies: Models of evaluation and quality of evidence. Evaluation 2(2): 195-2131.

Blundo-Canto G, Läderach P, Waldock J, Camacho K. 2017. Learning through monitoring, evaluation and adaptations of the "Outcome Harvesting" tool. Cah Agric 26(6): 65004. DOI: 10.1051/cagri/ 2017054.

Bornmann L, 2013. What is societal impact of research and how can it be assessed? A literature survey. Journal of the American Society for Information Science and Technology 64: 217-233.

Boutillier S, Laperche B. 2016. Christopher Freeman : la systémique de l'innovation. In: Burger-Helmchen T, Hussler C, Cohendet P, eds. Les grand auteurs du management de l'innovation et de la créativité. Condé-sur-Noireau : Éditions Management et Société.

Breumier P, Ramarosandratana A, Ramanantsoanirina A, vom Brocke K, Marquié C, Dabat M-H, et al. 2018. Évaluation participative des impacts de la recherche sur le riz pluvial d'altitude à Madagascar de 1980 à 2015. Cah Agric 27(1): 15004. DOI: 10.1051/cagri/2017065.

Cerdan C, Andrade N, Lima A, Vieira H, Silva E, Le Guerroué J-L. 2018. Indications géographiques et dynamiques collectives des territoires ruraux: quelle contribution de la recherche agricole? Cah Agric 27: 170081. DOI: 10.1051/cagri/2018013.

Clavel D, Gaye G. 2018. L'émergence de nouvelles coopératives semencières au Sénégal-Analyse de l'impact de la recherchedéveloppement sur l'arachide de 1999 à 2016. Cah Agric 27(1): 15008. DOI: $10.1051 /$ cagri/2017062.

Colinet L, Gaunand A, Joly P-B, Matt M. 2017. Des barèmes génériques pour évaluer les impacts de la recherche sur la société : l'exemple des impacts politiques. Cah Agric 26(6): 65006. DOI: 10.1051/cagri/2017056.

Dabat M-H, Grandjean A. 2018. La contribution de l'action publique à l'impact de la recherche. Cah Agric 27 : 15013. DOI: 10.1051/ cagri/2018003.

Donovan C. 2011. State of the art of assessing research impact: introduction to a special issue. Research Evaluation 20: 175-179.

Douthwaite B, Mayne J, McDougall C, Paz-Ybarnegaray R. 2017. Evaluating complex interventions: A theory-driven realist-informed approach. Evaluation 23(3): 294-311.

Ferré T, Medah I, Cruz J-F, Chtioui M, Dabat M-H, DevauxSpatarakis A. 2018. Innover dans le secteur de la transformation agroalimentaire en Afrique de l'Ouest. Cah Agric 27: 15011. DOI: 10.1051/cagri/2018004.

Gaunand A, Temple L, Trouche G. 2018. Évaluer les impacts des recherches en agriculture sur la société : outils, méthodes, études de cas. Cahiers Agricultures, numéro thématique. Disponible sur https://www.cahiersagricultures.fr/fr/component/toc/?task=topi c\&id $=872$.

Giraud G. 2018. L'innovation au service d'une agriculture durable, Postface. In : Faure G, Chiffoleau Y, Goulet F, Temple L, Touzard $\mathrm{JM}$, eds. Innovation et développement dans les systèmes agricoles et alimentaires. Montpellier: Éditions Quæ (sous presse).

Godin B, Vinck D. 2017. Critical studies of innovation. Cheltenham (UK): Edition Edward Elgar, 335 p.

Joly P-B, Gaunand A, Colinet L, Larédo P, Lemarié S, Matt M. 2015a. ASIRPA: A comprehensive theory-based approach to assessing the societal impacts of a research organization. Research Evaluation 24 (4): 1-14. DOI: 10.1093/reseval/rvv015.
Joly P-B, Colinet L, Gaunand A, Lemarié S, Laredo P, Matt M. 2015b. Évaluer l'impact sociétal de la recherche pour apprendre à le gérer : l'approche ASIRPA et l'exemple de la recherche agronomique. Annales des Mines-Gérer et Comprendre 122: 31-42.

Mayne J. 2012. Contribution analysis: Coming of age? Evaluation 18 (3): 270-280. DOI: 10.1177/1356389012451663.

Minkoua J-R, Temple L. 2018. L'offre de recherche converge-t-elle vers les besoins du secteur agroalimentaire au Cameroun ? Cah Agric 27: 170068. DOI: 10.1051/cagri/2018016.

Naudet JD, Delarue J, Bernard T. 2012. Évaluations d'impact: un outil de redevabilité ? Les leçons tirées de l'expérience de l'AFD. Revue d'Economie du Développement 26: 27-48.

Queste J, Wassenaar T. 2018. Comment la recherche concertée contribue à l'écologie territoriale. Cah Agric 27(1): 15006. DOI: $10.1051 /$ cagri/2017059.

Quiédeville S, Barjolle D, Stolze M. 2018. Using Social Network Analysis to evaluate the Impacts of the Research: On the transition to organic farming in the Camargue. Cah Agric 27: 15012. DOI: 10.1051/cagri/2018006.

Renkow M, Byerlee D. 2010. The impacts of CGIAR Research: A review of recent evidence. Food Policy 35(5): 391-402.

Ruegg R, Feller I. 2003. Toolkit for evaluating public R\&D investment: Models, methods, and findings from Atp's first decade. Editor Diane Pub Co. ISBN 10: 0756741599 / ISBN 13: 9780756741594.

Spaapen J, van Drooge L. 2011. Introducing "productive interactions" in social impact assessment. Research Evaluation 20: 211-218.

Stiglitz JE, Greenwald B. 2014. La nouvelle société de la connaissance. Paris : Éditions les liens qui libèrent, 443 p.

Temple L, Biénabe E, Barret D, Saint-Martin G. 2016. Methods for assessing the impact of research on innovation and development in the agriculture and food sectors. African Journal of Science, Technology, Innovation and Development 8(5-6): 399-410.

Temple L, Barret D, Blundo Canto G, Dabat MH, Devaux-Spatarakis A, Faure G, et al. 2018. Assessing impacts of agricultural research for development: A systemic model focusing on outcomes. Research Evaluation 27(2): 157-170. DOI: 10.1093/reseval/rvy005.

Toillier A, Devaux-Spartakis A, Faure G, Barret D, Marquié C. 2018. Comprendre la contribution de la recherche à l'innovation collective par l'exploration de mécanismes de renforcement de capacité. Cah Agric 27(1): 15002. DOI: 10.1051/cagri/2017055.

Touzard JM. 2018. L'innovation agricole et agroalimentaire au XxI siècle : maintien, effacement ou renouvellement de ses spécificités? In: Faure G, Chiffoleau Y, Goulet F, Temple L, Touzard JM, eds. Innovation et développement dans les systèmes agricoles et alimentaires. Montpellier : Éditions Quæ, pp. 41-57 (sous presse).

Touzard JM, Temple L, Faure G, Triomphe B. 2015. Innovation systems and knowledge communities in the agriculture and agrifood sector: a literature review. Journal of Innovation Economics and Management 2(17): 117-142.

Wasserman S. 1994. Social network analysis: Methods and applications. United Kingdom: Cambridge University Press, 852 p.

Weißhuhn P, Helming K, Ferretti J. 2017. Research impact assessment in agriculture - A review of approaches and impact areas. Research Evaluation 27(1): 36-42.

Wiek A, Talwar S, O'Shea M, Robinson J. 2014. Toward a methodological scheme for capturing societal effects of participatory sustainability research. Research Evaluation 23(2): 117-132.

Citation de l'article : Temple L, Gaunand A, Trouche G, Vall E. 2018. Évaluer les impacts des recherches en agriculture sur la société et les écosystèmes : outils, méthodes, études de cas. Cah. Agric. 27: 34002. 